\title{
INFLUENCE OF THE GRAVITATIONAL FIELD ON THE SHAPE OF SPECTRAL LINES IN SPECTRA OF SEYFERT GALAXIES AND QUASARS
}

\author{
L. Č. POPOVIĆ, I. VINCE and A. KUBIČELA \\ Astronomical Observatory, \\ Volgina 7, Belgrade \\ Yugoslavia
}

On the basis of preliminary examination of the model of Seyfert galaxies and quasars we have concluded that their gravitational field may have an important influence on the shape of spectral lines when the emission cloud is in gravitational field of massive nuclei (mass about $10^{7}-10^{8} \mathrm{M}_{\odot}$ for Seyfert galaxies, see e.g. Padovani et al. 1990), and the emission cloud is large enough (for example about $10^{14}-10^{15} \mathrm{~m}$ for Broad Line Region (BLR) of NGC 7469 (Bonatto \& Pastoriza 1990) or about $10^{21} \mathrm{~m}$ for quasar 3C 257 (Forbes et al. 1990)), so that the emitters are in very different gravitational field along the line of sight.

In this preliminary analyses we consider a very simple model of Seyfert galaxy, in wich a massive nuclei is surrounded by circumnucleus cloud with only one sort of emitters and with only one possible transition $m \rightarrow n$. The circumnucleus cloud is static and opthicaly thin. We assume that the profile of spectral line, at a given point of the cloud is Lorentzian one.

If we consider that the emitters are in the gravitational field of a mass $M$ at a distance $r$ from the central body, and that the gravitational potential at observer is negligibly small, the transition frequency $\left(\omega_{0}\right)$ will be shifted by $\omega^{\prime}-\omega_{0}=\omega_{0} G M /\left(r c^{2}\right)$ (see e.g. Weinberg 1972), where $G$ is the gravitational constant and $\omega^{\prime}$ is the shifted frequency. For a cloud of thickness $D=R-R_{0}$ the emerging line profile is given as a sum of radiation of all emitters along the line of sight. In this case intensity at a given frequency in shape of spectral line profile can be given by

$$
S^{\prime}\left(\omega, R, R_{0}, M\right)=S(\omega) \Phi\left(\omega, R_{0}, R, M\right),
$$

where $S$ is the Lorenzian profile and $\Phi$ is the correction function that determines the degree of spectral line profile distortion produced by the gravitation field.

As an illustration of the influence of gravitational field on spectral line shape we calculated the shape of $H_{\alpha}$ line with the following parameters: $M=10^{8} \mathrm{M}_{\odot}, R_{0}=10^{14} \mathrm{~m}, R=10^{16} \mathrm{~m}$. As a results we have obtained that the spectral line is shifted by about $0.12 \AA$ toward the red and is broaded about $18 \%$. The blue wing of the deformed spectral line is lower, while the red one is higher than in the unperturbed line profile. This influence is more prominent for spectral lines in infrared and radio than in ultraviolent range of wavelength.

\section{REFERNCES}

Bonatto, C. J., and Pastoriza, M. G., 1990, ApJ. 353, 445.

Padovani, P., Burg, R., and Edelson, R. A., 1990, ApJ. 353, 438.

Forbes, D. A., Crawford, C. S., Fabian, A. C. \& Johnstone, R. M., 1990, Mon. Not. R. Astr. Soc. 244, 680 .

Weinberg, S., 1972, Gravitation and Cosmology, John Wiley and Sons, New York - London - Sydney - Toronto. 